\title{
The powerful high pressure tool for protein conformational studies
}

\section{S. Marchal ${ }^{1}$, J. Torrent ${ }^{1}$, P. Masson ${ }^{2}$, J.M. Kornblatt ${ }^{3}$, P. Tortora ${ }^{4}$, P. Fusi ${ }^{4}$, \\ R. Lange ${ }^{1}$ and \\ C. Balny ${ }^{1}$}

Abstract

The pressure behavior of proteins may be summarized as a the pressure-induced disordering of their structures. This thermodynamic parameter has effects on proteins that are similar but not identical to those induced by temperature, the other thermodynamic parameter. Of particular importance are the intermolecular interactions that follow partial protein unfolding and that give rise to the formation of fibrils. Because some proteins do not form fibrils under pressure, these observations can be related to the shape of the stability diagram. Weak interactions which are differently affected by hydrostatic pressure or temperature play a determinant role in protein stability. Pressure acts on the $2^{\circ}, 3^{\circ}$ and $4^{\circ}$ structures of proteins which are maintained by electrostatic and hydrophobic interactions and by hydrogen bonds. We present some typical examples of how pressure affects the tertiary structure of proteins (the case of prion proteins), induces unfolding (ataxin), is a convenient tool to study enzyme dissociation (enolase), and provides arguments to understand the role of the partial volume of an enzyme (butyrylcholinesterase). This approach may have important implications for the understanding of the basic mechanism of protein diseases and for the development of preventive and therapeutic measures.

\section{Introduction}

Recent results have demonstrated the potential of high hydrostatic pressure methods to reveal various protein structural changes that are inaccessible to conventional biochemical or biophysical methods $(1,2)$. The native structure of a protein, i.e., the conformation that displays biological activities, is the result of a delicate balance between stabilizing and destabilizing interac-
Key words

- High pressure

- Prion protein

- Amyloid

- Ataxin

- Enolase

- Butyrylcholinesterase

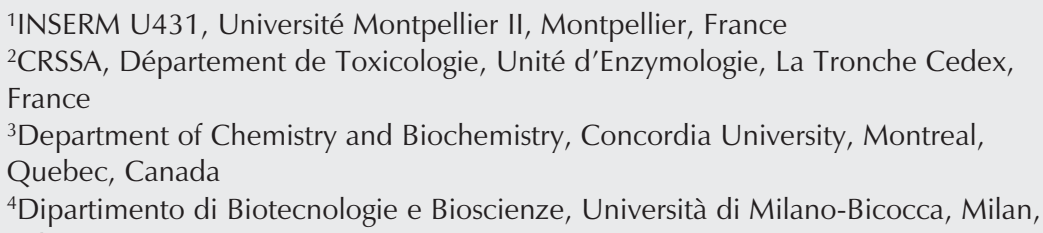


main reason for this are weak interactions which are involved in the three-dimensional structure of biomacromolecules and in biocatalytic processes. Weak interactions are one of the keys to understanding the effects of pressure on biomaterials since these materials are strongly affected by pressure (and also by temperature) (4).

\section{The role of weak interactions and their pressure dependency}

The biological role of weak interactions has been already described in the literature (5), and in the current paper we would like to present recent examples of some biological reactions in which such interactions are affected by pressure.

Weak interactions are involved either at the intramolecular or the intermolecular level. The main intramolecular interactions found in biomacromolecules are ionic, hydrophobic, hydrogen interactions, and hydration $(\mathrm{H}$ bonds with water molecules of the first hydration shell and with water trapped in protein cavities). Intermolecular interactions are those governing protein-protein, enzymesubstrate or enzyme-inhibitor, protein-ligand, and protein-solvent interactions. Depending on their nature, these interactions are positively or negatively pressure-affected (and/ or temperature-affected).

Electrostatic interactions are weakened at elevated pressure due to electrostriction. Hydrogen bonds, as well as stacking of aromatic residues are stabilized by high pressure, whereas hydrophobic interactions, which are the most important weak interactions for protein stabilization, are unfavorably affected by pressure (6).

Both intra- and intermolecular interactions are involved in reaction pathways. This is the case of protein aggregation where intramolecular interactions govern the first steps of the reaction, i.e., the transformation of a globular native protein conformation into a partially unfolded state (a reaction where one or more intermediate states could be involved). The second step of the aggregation processes is then governed by intermolecular reactions occurring where stacking between proteins leads to aggregates, e.g., amyloid (or fiber) formation (7).

Covalent interactions are out of the scope of this review because they are not pressure dependent below $1 \mathrm{GPa}$. However, cases have been reported in which even higher pressures have been applied.

\section{Pressure effects on protein structures}

If the primary protein structures are generally unaffected by pressure at ordinary temperature since they are maintained by covalent interactions, secondary, tertiary and quaternary structures are affected by pressure because weak interactions are involved. Quaternary structures are more affected than the tertiary and secondary structures. Recent reviews deal with this problem $(8,9)$.

\section{Typical examples}

The following results were obtained mainly using spectroscopic methods specially adapted for high pressure experiments $(10,11)$.

\section{Pressure effects on tertiary structure:} the case of prion proteins

According to Prusiner's hypothesis, prion protein $(\mathrm{PrP})$ is involved in transmissible spongiform encephalopathy diseases (12). This appears to be caused by an aberrant conformational change of the cellular PrP, leading to its deposition in the form of amyloid fibrils. Recently, we have shown that using high-pressure perturbation, recombinant PrP can be converted to different novel misfolded conformers (13-15). By a judicious choice of pressure, temperature, $\mathrm{pH}$, and incubation time, it is possible to attain 
several distinct PrP structures. Two of them are potentially malignant: one appears to be a pre-amyloid aggregated form, and the other is an amyloid aggregate characterized by the formation of fibrils and fibrillar bundles. Both aggregate types were resistant to protein kinase digestion. Moreover, the amyloid fibrils showed birefringence of polarized light after Congo red staining, a strongly decreased 1-anilinonaphthalene-8-sulfonic acid (ANS) binding capacity and an enhanced binding of thioflavin T. This behavior suggested that pressure unraveled alternative routes of PrP misfolding in comparison with classical denaturant agents such as urea or elevated temperature.

It is becoming increasingly clear that protein aggregation is a complex process involving several kinds of intermediates and resulting in different amyloid fibrils or amorphous aggregates (16). Our results $(14,15)$ suggest that during recombinant PrP misfolding and aggregation, induced by different perturbing agents (i.e., chemical denaturants at acidic $\mathrm{pH}$, high temperature, or high pressure), competing pathways exist leading to a mixture of different conformations (i.e., amyloid and amorphous aggregates). Depending on the experimental conditions, different routes of misfolding can be favored, leading to various final conformers. Indeed, different structural responses to pressure are observed between the different PrP aggregates formed. We demonstrated that aggregates resulting from heating above $60^{\circ} \mathrm{C}$ can be completely reversed by high pressure. In contrast, we monitored only partial solubilization under high pressure of the aggregates formed at $1 \mathrm{M} \mathrm{GdnHCl}$ and $\mathrm{pH}$ 4.0. In addition, within the experimental resolution of our spectroscopic techniques, aggregates obtained after overnight incubation at $600 \mathrm{MPa}$ were insensitive to a further pressurization cycle. Such complex multitude of different PrP isoforms obtained is in accordance with branching pathways during aggregation. One of them could be involved in populating the pathologic prion form. Thus, we are pursuing our efforts to attain the infectious conformation from a recombinant PrP. We may speculate, in agreement with the latest findings from Prusiner's group, that the infectious form may represent a restricted fraction of $\beta$-rich forms produced, not sufficient to trigger disease (17).

Increasing protein concentration increases the likelihood of aggregation. For instance, at low protein concentration, a simple two-state, reversible unfolding transition of recombinant PrP was observed. The results suggested that at high pressure (between 400-600 MPa), complete unfolding of $\mathrm{PrP}$ was not achieved. It was apparent that the tyrosine residues were, on average, more exposed to the aqueous solvent, and the formerly two well-exposed tryptophans displayed interactions with nonpolar residues. Although there was no evidence of the appearance of scattering aggregates at high pressure, reversible thioflavin $\mathrm{T}$ binding was observed, suggesting a structural change from a misfolded to a metastable oligomeric structure. To investigate further the origin of this enhanced thioflavin-T binding at high pressure, we performed unfolding at a higher protein concentration $(2 \mathrm{mg} / \mathrm{ml})$. After treatment of PrP with pressures above $500 \mathrm{MPa}$, the above-mentioned thioflavin T-binding intermediate underwent aggregation.

In the case of protein-coding mutations, the relevance of specific protein domains to the tendency to aggregate can be tested. The high-pressure approach applied to singlepoint variants of the 132-160 region revealed an ensemble of conformations between native and unfolded states of $\operatorname{PrP}(18)$. The results argue in favor of a central, eventually initial role of the 132-160 region in the $\mathrm{PrP}^{\mathrm{C}}$ to $\mathrm{PrP}^{\mathrm{Sc}}$ conversion, and the significant structural stabilizing role of two particular residues. In fact, depending on the experimental conditions used, the pressure-induced intermediate states of the variant proteins underwent aggregation. In addition, the variants 
were characterized by a substantially increased propensity to form a $B$-sheet-rich conformer under identical conditions, when compared to wild-type protein.

\section{Pressure-induced unfolding of human ataxin-3}

The human ataxin-3, a member of the polyglutamine family of proteins, is responsible for spinocerebellar ataxia type 3 , also known as Machado-Joseph disease (19). Expansion of a CAG repeat coding for polyglutamine (polyGln) in the C-terminal part of the $42-\mathrm{kDa}$ ataxin-3 protein leads to neurodegenerative disorders, as well as formation of proteinaceous aggregates such as nuclear inclusions (20). Although the elongated polyGln stretch of mutant ataxin-3 is considered to be the pathological hallmark of Machado-Joseph disease and protein fibrillogenesis (21), non-expanded forms of ataxin-3 are also observed within nuclear inclusions in neurons (22). In order to explore how perturbation of the folding process leads to inappropriate structural change and to gain further insight into the modulation of protein stability by the polyGln tract, we performed a comparative analysis of two non-pathological variants of ataxin-3, the human protein with 26 glutamines (Q26) within its polyGln tract and the murine orthologue containing only 6 Gln residues (Q6). These proteins display high sequence homology with a conserved N-terminal region (Josephin domain) and a C-terminal region where the polyGln sequence is located.

Using complementary biophysical techniques including spectroscopic methods and electron microscopy, we have shown that the pressure-induced unfolding of human ataxin-3 (Q26) was characterized by a complex mechanism, implying several steps and unfolding intermediates within the experimental pressure range (23). First, the protein started to unfold in a relatively low-pressure range $\left(\mathrm{P}_{1 / 2}=145 \mathrm{MPa}\right)$. The destabilizing effect of pressure induces a partial solvent exposure of tryptophan residues and hydrophobic regions of the protein (conformer 1). Based on various results, we proposed that the length of the polyGln tract disrupts the stability of the native conformation of human ataxin-3. Perturbation by higher pressure (600 MPa) leads to subsequent conformational changes of Q26 protein and to the accumulation of a partially unfolded, aggregation prone species (conformer 2). In addition, thioflavin $\mathrm{T}$ bound to the partly unfolded state of ataxin-3 suggesting the formation of soluble metastable structures at high pressure. This conformer underwent concentration-dependent aggregation that probably followed a nucleation-dependent pathway, as observed by electron microscopy of ataxin-3 aggregates.

In order to validate our proposed mechanism of human Q26 ataxin-3 unfolding and aggregation for pathogenic forms of ataxin3 , we used as a model the human Q36 variant which forms amyloid fibrils at high temperature (24). Our data show at least two distinct unfolded conformers of the expanded form of ataxin-3 accumulated during pressurization (25), similar to those observed for the human Q26 ataxin-3 (23). The only major difference between the pressure-induced unfolding profiles of Q26 and Q36 proteins was the amplitude of the first transition. However, conformational changes occurring at a later stage were similar for both proteins. This observation supports the notion that even if elongation of the polyglutamine tract in ataxin-3 affects the native conformation of the protein, non-polyglutamine regions and domains remain less sensitive to unfolding at moderate pressure.

Altogether, these results are consistent with the hypothesis that the glutamine repeat length is a negative determinant of ataxin-3 stability but not the only factor determining protein aggregation (26). The thermodynamic stability of human ataxin-3 is mainly 
governed by the properties of the Josephin domain (27). Variations of glutamine repeat length increase the protein's propensity to aggregate as a distinct pathway with respect to protein unfolding. In order to assess our knowledge of ataxin-3 fibrillogenesis and to define the structural properties of the amyloidogenic intermediate, further investigations are currently underway using hydrostatic pressure as a thermodynamic perturbant.

\section{Pressure as a tool to study enzyme dissociation}

This example concerns experiments performed with yeast enolase to try to answer the following question: can monomers of yeast enolase have enzymatic activity?

Enolase catalyzes the interconversion of 2-phosphoglyceric acid and phosphoenolpyruvate. Most enolases are dimers. Each dimer contains two active sites and the active site appears to be completely contained within the monomer (28-30). This suggests that enolase monomers should be active. However, in most dissociation experiments on enolase, the resulting monomers were inactive (31-35). As a general rule, the pressure required to dissociate oligomeric proteins is less than that required for denaturation. We felt, therefore, that it was reasonable to hope that hydrostatic pressure would dissociate enolase, while preserving the native secondary and tertiary structure and the enzymatic activity.

However, our first experiments with hydrostatic pressure proved us wrong. We subjected yeast enolase to pressure and monitored the ultraviolet (UV) spectrum, activity after release of pressure and, using stoppedflow under pressure, activity under pressure. The changes in UV and activity were fully reversible and all occurred in the same pressure range, with no evidence of intermediates. The pressure-induced inactivation of enolase was a two-state process. The mono- mers were inactive. In the same set of experiments, we determined the effects of high salt $(\mathrm{KCl})$ and EDTA on the dissociation. Enolase is a metalloenzyme and our experiments were normally performed in the presence of $\mathrm{Mg}^{2+}$. The results showed that at least three processes were involved in dissociation of enolase by pressure: disruption of electrostatic interactions, loss of $\mathrm{Mg}^{2+}$, and exposure and hydration of buried surfaces (36).

Could we, by using a different divalent cation, produce holo-monomers (monomers that still had the cation bound)? If so, would they be active? Preliminary experiments comparing the effects of pressure on apo-enolase, $\mathrm{Mg}^{2+}$-enolase, and $\mathrm{Mn}^{2+}$-enolase indicated that the $\mathrm{Mn}^{2+}$ stayed bound to the protein at pressures up to $220 \mathrm{MPa}$. Detailed spectral studies with $\mathrm{Mn}^{2+}$-enolase showed that pressure was producing multiple changes in the fluorescence and UV spectra, similar to those produced with $\mathrm{Mg}^{2+}$-enolase. However, with $\mathrm{Mn}^{2+}$-enolase, the spectral changes did not all occur at the same pressure, indicating that multiple processes are occurring. Experiments were then performed as a function of protein concentration. They showed that the first step to occur was dissociation. The spectral changes for this step were small; simulations showed that the spectral changes are due to changes in the polarity of the environment of several tyrosines. We believe that these changes arise from two tyrosines that are pointing at a cleft between the two subunits (37). In this pressure range where the first process occurs, we could not demonstrate inactivation of the enzyme. Pressure has produced monomers that have retained the divalent cation, retained enzymatic activity and, based on the small spectral changes, have retained the native structure (38).

As the pressure is increased, further changes occur in both the UV and fluorescence spectra. At least some of these are due to changes in the environment of tryptophan 
residues. Depending on its concentration, $\mathrm{Mn}^{2+}$ may be lost or retained. Although further spectral changes are occurring, as long as $\mathrm{Mn}^{2+}$ remains bound, the monomers are active. With hydrostatic pressure, we are able to produce and study three conformations of this enzyme: apo-monomers, $\mathrm{Mn}^{2+}$ monomers (low pressure form), and $\mathrm{Mn}^{2+}$ monomers (high pressure form).

We would like to learn something about the structural differences between these forms of the protein and how the changes in structure relate to changes in activity. There is a tryptophan - W56 - that is at the subunit interface. The side chain points to the interior of the subunit, while the backbone amide is hydrogen bonded to the side chain of a glutamic acid residue on the other subunit. We have used mutagenesis to perturb this subunit interaction. In the low pressure $\mathrm{Mn}^{2+}$ monomers there are no changes in tryptophan fluorescence, indicating that the side chain is still buried in the interior (Padovani A and Kornblatt MJ, unpublished data). As the pressure is increased, this residue becomes more exposed. Residue 56 is at the end of a long loop, residues 36-60. Near the beginning of this loop is a very mobile region, residues 37-41, which changes conformation upon binding of substrate. It is thus possible that the movement of W56 which occurs in the monomer as the pressure is raised is communicated to the active site via this loop.

We have succeeded in producing multiple conformations of yeast enolase by the application of hydrostatic pressure. The combination of spectral and activity measurements under pressure and site-directed mutagenesis will allow us to begin to dissect the structural and functional changes that are occurring in this protein.

Role of the partial molar volume of proteins. The case of an enzyme

Butyrylcholinesterase (BuChE, EC. 3.1.1.8), a non-specific esterase, is closely related to acetylcholinesterase (EC.3.1.1.7), the enzyme which terminates the action of the neurotransmitter acetylcholine. BuChE has no clear physiological function, but may be involved in the development of the nervous system and in neurodegenerative diseases. However, it scavenges or detoxifies numerous poisonous esters (such as organophosphates, carbamates, and cocaine). The $\mathrm{X}$-ray structure of human $\mathrm{BuChE}$ was recently solved (39). Precise knowledge of the catalytic mechanisms of BuChE and of structure-activity relationships are needed. Several kinetic studies of BuChE under pressure have already been published. These studies provided valuable information on physicochemical events accompanying substrate/inhibitor binding and chemical steps along catalytic pathways. In particular, the importance of hydration changes during BuChEcatalyzed reactions was brought to the fore (40-42). An important issue is to understand the molecular mechanisms underlying the different reactivity of BuChE with oxo-esters versus thio-esters. Because pressure is known to alter the elementary rate constants, depending on the sign and magnitude of volume changes and activation volumes accompanying each step in an enzyme reaction (43), the effects of pressure on the steadystate catalytic parameters of human BuChE towards two positively charged substrates were investigated. Substrates were the oxoester benzoylcholine ( $\mathrm{BzCh})$ and the thioester benzoylthiocholine (BzSCh). Kinetic measurements were achieved using the stopped-flow apparatus working at a pressure up to $200 \mathrm{MPa}$ (10). Under standard conditions, the rate-limiting step for hydrolysis of $\mathrm{BzCh}$ by human $\mathrm{BuChE}$ is deacylation $\left(\mathrm{k}_{3}\right)$, whereas it is acylation $\left(\mathrm{k}_{2}\right)$ for hydrolysis of the homologous thio-ester BzSCh (44). Steady-state hydrolysis of $\mathrm{BzCh}$ and $\mathrm{BzSCh}$ by wild-type $\mathrm{BuChE}$ and its $\mathrm{D} 70 \mathrm{G}$ mutant was investigated at different hydrostatic pressures (up to $150 \mathrm{MPa}$ ) (45). Elementary volumetric contributions, i.e., volume 
changes and activation volumes upon initial binding, productive binding, acylation, and deacylation were calculated from the pressure differentiation of binding and kinetic constants. This analysis shed light on the molecular events taking place along the pathways of $\mathrm{BzCh}$ and $\mathrm{BzSCh}$ hydrolysis by $\mathrm{BuChE}$. Indeed, a differential nonlinear pressure dependence of the catalytic parameters $\left(K_{\mathrm{m}}, k_{\mathrm{cat}}\right.$ and $\left.k_{\mathrm{cat}} / K_{\mathrm{m}}\right)$ for hydrolysis of both substrates was shown. Non-linearity of the plots may be explained in terms of compressibility changes or rate-limiting changes with pressure. To distinguish between these two possibilities, irreversible enzyme phosphorylation by diisopropylfluorophosphate was studied in the presence of substrate (BzSCh) under pressure. Phosphorylation of the enzyme was similar to acylation by esters/thio-esters $\left(\mathrm{k}_{2}\right)$. There was no pressure dependence of volume change for diisopropylfluorophosphate binding or activation volume for phosphorylation, indicating no compressibility change with pressure. Thus, analysis of the pressure dependence for steady-state hydrolysis of substrates, and for an irreversible acylation reaction provided evidence that no enzyme compressibility changes occurred during BuChE-catalyzed reactions, and that the nonlinear pressure dependence of substrate hydrolysis reflects changes in the rate-limiting step with pressure. A change in the rate-determining step occurred at a pressure of $100 \mathrm{MPa}$ for hydrolysis of $\mathrm{BzCh}$ by BuChE. For hydrolysis of BzSCh the change occurred at higher pressures because $\mathrm{k}_{2}<<\mathrm{k}_{3}$ at atmospheric pressure for this substrate.

Though activation volumes are related to the activation free energy change by the relation $\left(\delta \Delta \mathrm{G}^{\ddagger} / \Delta \mathrm{P}\right)_{\mathrm{T}}=\Delta \mathrm{V}^{\ddagger}$, the sign and magnitude of $\Delta \mathrm{V}^{\ddagger}$ did not provide enough information to characterize the rate-determining step at atmospheric pressure in terms of binding energy and geometry of the transition state. However, analysis of volume profiles can provide new insights into the fluctuations of the enzyme volumes along the catalytic pathway. Such an approach was successfully used for other enzyme systems (46). Concerning the molecular bases of the differential reactivity of $\mathrm{BuChE}$ towards oxoesters versus thio-esters, the activation volume for acylation was found to be positive for $\mathrm{BzCh}$, whereas it was negative for $\mathrm{BzSCh}$. This indicates that the transition state for acylation by $\mathrm{BzSCh}$ is more compressed compared to that for $\mathrm{BzCh}$. This difference between thio- and oxo-ester may result from electronic and/or steric features of the ethereal sulfur atom compared to oxygen. Study on other couples of oxo/thiosubstrates is in progress in order to determine the relative contributions of heteroatom electro-negativity and bond geometry to the stabilization of acylation transition state.

Through the examples above we have shown that pressure provides convenient means to study protein conformational changes. Recent results from different laboratories indicate that pressure is particularly applicable to the study of protein aggregation and/or amyloid fibril formation. This topic is a challenge for the understanding of amyloidoses which are a variety of diseases or pathological states that share the common property of b-structured protein fibril deposits (amyloids). High-pressure experiments have been performed using both model and pathological proteins (47-51). As pointed out recently by Foguel and Silva (52): "The use of high pressure promises to contribute to the identification of the mechanism behind these effects and the creation of therapies against these diseases".

\section{Acknowledgments}

We wish to express our sincere thanks to Drs. J.-P. Liautard and M.T. Alvarez-Martinez (U 432 INSERM, Montpellier, France) who contributed to the prion results and to N. Bec (E229 INSERM, Montpellier, France) for the $\mathrm{BuChE}$ high-pressure experiments. 


\section{References}

1. Silva JL, Foguel D \& Royer CA (2001). Pressure provides new insights into protein folding, dynamics and structure. Trends in Biochemical Sciences, 26: 612-618.

2. Balny C, Masson P \& Heremans K (Editors) (2002). Frontiers in High-Pressure Biochemistry and Biophysics. Elsevier, Amsterdam, The Netherlands.

3. Mozhaev VV, Heremans K, Frank J et al. (1996). High pressure effects on protein structure and function. Proteins: Structure, Function, and Genetics, 24: 81-91.

4. Silva JL \& Weber G (1993). Pressure stability of proteins. Annual Review of Physical Chemistry, 44: 89-113.

5. Gross M \& Jaenike R (1994). Proteins under pressure. The influence of high hydrostatic pressure on structure, function and assembly of proteins and protein complexes. European Journal of Biochemistry, 221: 617-630.

6. Balny C (2004). Pressure effects on weak interactions in biological systems. Journal of Physics: Condensed Matter, 16: S1245-S1253.

7. Fink AL (1998). Protein aggregation: folding aggregates, inclusion bodies and amyloid. Folding and Design, 3: R9-R23.

8. Boonyaratanakornkit BB, Park CB \& Clark DS (2002). Pressure effects on intra- and intermolecular interactions within proteins. Biochimica et Biophysica Acta, 1595: 235-249.

9. Heremans K \& Smeller L (1998). Protein structure and dynamics at high pressure. Biochimica et Biophysica Acta, 1368: 353-370.

10. Balny C, Saldana JL \& Dahan N (1984). High-pressure stopped-flow spectrometry at low temperature. Analytical Biochemistry, 139: 178189.

11. Lange R, Frank J, Saldana JL et al. (1996). Fourth derivative UVspectroscopy of proteins under high pressure. European Biophysics Journal, 24: 277-283.

12. Prusiner SB, Scott MR, DeArmond SJ et al. (1998). Prion protein biology. Cell, 93: 337-348.

13. Alvarez-Martinez MT, Torrent J, Lange R et al. (2003). Optimized overproduction, purification, characterization and high pressure sensitivity of the prion protein in the native ( $\mathrm{PrP}^{\mathrm{c}}$-like) or amyloid ( $\mathrm{PrPsc}_{-}$ like) conformation. Biochimica et Biophysica Acta, 1645: 228-240.

14. Torrent J, Alvarez-Martinez MT, Heitz F et al. (2003). Alternative prion structural changes revealed by high pressure. Biochemistry, 42: 1318-1325.

15. Torrent J, Alvarez-Martinez MT, Harricane MC et al. (2004). High pressure induces scrapie-like prion protein misfolding and amyloid formation. Biochemistry, 43: 7162-7170.

16. Randolph TW, Seefeldt M \& Carpenter JF (2002). High hydrostatic pressure as a tool to study protein aggregation and amyloidosis. Biochimica et Biophysica Acta, 1595: 224-234.

17. Legname G, Baskakov IV, Nguyen $\mathrm{HO}$ et al. (2004). Synthetic mammalian prions. Science, 305: 673-676.

18. Torrent J, Alvarez-Martinez MT, Liautard JP et al. (2005). The role of the $132-160$ region in prion protein conformational transitions. Protein Science, 14: 956-967.

19. Kawaguchi $Y$, Okamoto T, Taniwaki M et al. (1994). CAG expansions in a novel gene for Machado-Joseph disease at chromosome 14q32.1. Nature Genetics, 8: 221-228.

20. Perutz MF, Johnson T, Suzuki M et al. (1994). Glutamine repeats as polar zippers: their possible role in inherited neurodegenerative diseases. Proceedings of the National Academy of Sciences, USA, 91: 5355-5358.

21. Fujigasaki H, Uchihara T, Koyano S et al. (2000). Ataxin-3 is translo- cated into the nucleus for the formation of intranuclear inclusions in normal and Machado-Joseph disease brains. Experimental Neurology, 165: 248-256.

22. Paulson HL, Perez MK, Trottier $Y$ et al. (1997). Intranuclear inclusions of expanded polyglutamine protein in spinocerebellar ataxia type 3. Neuron, 19: 333-344.

23. Marchal S, Shehi E, Harricane MC et al. (2003). Structural instability and fibrillar aggregation of non-expanded human ataxin-3 revealed under high pressure and temperature. Journal of Biological Chemistry, 278: 31554-31563.

24. Shehi E, Fusi P, Secundo F et al. (2003). Temperature-dependent, irreversible formation of amyloid fibrils by a soluble human ataxin-3 carrying a moderately expanded polyglutamine stretch (Q36). Biochemistry, 42: 14626-14632.

25. Torrent J, Marchal S, Tortora P et al. (2004). High pressure, an alternative approach to understand protein misfolding diseases. Cellular and Molecular Biology (Noisy-Le-Grand), 50: 377-385.

26. Chow MK, Ellisdon AM, Cabrita LD et al. (2004). Polyglutamine expansion in ataxin-3 does not affect protein stability: implications for misfolding and disease. Journal of Biological Chemistry, 279: 47643-47651.

27. Masino L, Nicastro G, Menon RP et al. (2004). Characterization of the structure and the amyloidogenic properties of the Josephin domain of the polyglutamine-containing protein ataxin-3. Journal of Molecular Biology, 344: 1021-1035.

28. Stec B \& Lebioda $L$ (1990). Refined structure of yeast apo-enolase at $2025 \AA$ A resolution. Journal of Molecular Biology, 211: 235-248.

29. Zhang E, Brewer JM, Minor W et al. (1997). Mechanism of enolase: the crystal structure of asymmetric dimer enolase-2-phospho-Dglycerate/enolase-phosphoenolpyruvate at $2.0 \AA$ resolution. Biochemistry, 36: 12526-12534.

30. Wedekind JE, Poyer RR, Reed GH et al. (1994). Chelation of serine 39 to $\mathrm{Mg}^{2+}$ latches a gate at the active site of enolase: structure of the bis $\left(\mathrm{Mg}^{2+}\right)$ complex of yeast enolase and the intermediate analog phosphonoacetohydroxamate at 2.1 Å resolution. Biochemistry, 33: 9333-9342.

31. Brewer JM, Faini GJ, Wu CA et al. (1978). Characterization of the subunit dissociation of yeast enolase. In: Catsimpoolas N (Editor), Physical Aspects of Protein Interactions. Elsevier/North-Holland, Amsterdam, The Netherlands, 57-78.

32. Trepannier D, Wong C \& Kornblatt MJ (1990). The salt-induced dissociation and inactivation of a mammalian enolase: evidence for the formation of active monomers. Archives of Biochemistry and Biophysics, 283: 271-277.

33. Kornblatt MJ, Al-Ghanim A \& Kornblatt JA (1996). The effects of sodium perchlorate on rabbit muscle enolase: spectral characterization of the monomer. European Journal of Biochemistry, 236: 78-84.

34. Marangos PJ, Parma AM \& Goodwin FK (1978). Functional properties of neuronal and glial isoenzymes of brain enolase. Journal of Neurology, 31: 727-732.

35. Chorazyczewski J, Danielewicz R \& Wolny M (1987). Immobilization of pig muscle enolase. Studies on the activity of subunits. Acta Biochimica Polonica, 34: 421-430.

36. Kornblatt MJ, Lange R \& Balny C (1998). Can monomers of yeast enolase have enzymatic activity? European Journal of Biochemistry, 251: 775-780.

37. Kornball JA, Kornblatt MJ \& Hui Bon Hoa G (1995). Second derivative spectroscopy of enolase at high hydrostatic pressure: an ap- 
proach to the study of macromolecular interactions. Biochemistry, 34: 1218-1223.

38. Kornblatt MJ, Lange R \& Balny C (2004). Use of hydrostatic pressure to produce 'native' monomers of yeast enolase. European Journal of Biochemistry, 271: 3897-3904.

39. Nicolet $Y$, Lockridge $O$, Masson $P$ et al. (2003). Crystal structure of human butyrylcholinesterase and of its complexes with substrate and products. Journal of Biological Chemistry, 278: 41141-41147.

40. Masson P \& Balny C (1990). Conformational plasticity of butyrylcholinesterase as revealed by high pressure experiments. Biochimica et Biophysica Acta, 1041: 223-231.

41. Masson P, Cléry C, Guerra P et al. (1999). Hydration change during the aging of phosphorylated human butyrylcholinesterase: importance of residues aspartate- 70 and glutamate-197 in the water network as probed by hydrostatic and osmotic pressures. Biochemical Journal, 343: 361-369.

42. Masson P, Froment MT, Fort S et al. (2002). Butyrylcholinesterasecatalyzed hydrolysis of $\mathrm{N}$-methylindoxyl acetate: analysis of volume changes upon reaction and hysteresis behavior. Biochimica et Biophysica Acta, 1595: 229-243.

43. Morild E (1981). The theory of pressure effects on enzymes. Advances in Protein Chemistry, 34: 93-166.

44. Masson P, Golstein BN, Debouzy JC et al. (2004). Damped oscillatory hysteretic behaviour of butyrylcholinesterase with benzoylcholine as substrate. European Journal of Biochemistry, 271: 220-234.

45. Masson P, Bec N, Froment MT et al. (2004). Rate-determining step of butyrylcholinesterase-catalyzed hydrolysis of benzoylcholine and benzoylthiocholine. European Journal of Biochemistry, 271: 19801990.

46. Gekko K (2002). Compressibility gives new insight into protein dynamics and enzyme function. Biochimica et Biophysica Acta, 1595: 382-386.

47. Smeller L, Rubens P \& Heremans K (1999). Pressure effect on the temperature-induced unfolding and tendency to aggregate of myoglobin. Biochemistry, 38: 3816-3820.

48. Dzwolak W, Ravindra R, Lendermann J et al. (2003). Aggregation of bovine insulin probed by DSC/PPC calorimetry and FTIR spectroscopy. Biochemistry, 42: 11347-11355.

49. Kuwata K, Li H, Yamada H et al. (2002). Locally disordered conformer of the hamster prion protein: a crucial intermediate to PrPSc? Biochemistry, 41: 12277-12283.

50. De Felice FG, Vieira MNN, Meirelles MNL et al. (2004). Formation of amyloid aggregates from human lysozyme and its disease-associated variants using hydrostatic pressure. FASEB Journal, 18: 10991101.

51. Martins SM, Chapeaurouge A \& Ferreira ST (2003). Folding intermediates of the prion protein stabilized by hydrostatic pressure and low temperatures. Journal of Biological Chemistry, 278: 5044950455.

52. Foguel D \& Silva JL (2004). New insights into the mechanisms of protein misfolding and aggregation in amyloidogenic diseases derived from pressure studies. Biochemistry, 43: 11361-11370. 